\title{
„Psychotherapie im Tageszentrum für Borderlinestörung“ Institut für Psychotherapie mit Tageszentrum für Borderlinestörung, Psychosoziale Dienste in Wien
}

\author{
Susanne Margreiter
}

Online publiziert: 21. Oktober 2020

(C) Der/die Autor(en) 2020

Zusammenfassung Dieser Artikel beschäftigt sich mit der Behandlung von Patient_innen mit der Diagnose „Borderline Persönlichkeitsstörung“ in einem ambulanten, therapeutischen Setting. Seit September 2009 Jahren bieten wir im Tageszentrum des Instituts für Psychotherapie des PSD Wien (Psychosoziale Dienste Wien) ein intensives, störungsspezifisches, verhaltenstherapeutisch basiertes Therapieprogramm über 12 Wochen für betroffene Patient_innen an. Tagesklinische Angebote haben viele Vorteile, z. B. den direkteren Transfer des Gelernten in den Alltag, erfordern aber auch viel Flexibilität und Unmittelbarkeit in der Behandlung.

Patient_innen, die an einer Borderline Störung erkrankt sind, benötigen, je nach Schweregrad der Erkrankung, häufig ein sehr komplexes Behandlungsangebot, das im Rahmen einer spezialisierten Institution leichter angeboten werden kann, als in Einzelpraxen. Als psychotherapeutische Einrichtung des PSD Wien sind wir gut vernetzt und können auch im Krisenfall schnell und effizient handeln. Die Versorgungssituation betroffener Patient_innen in Österreich ist prekär, die ambulanten Angebote für diese Patient_innengruppe sind nicht in ausreichender Menge vorhanden.

Generell stellt die psychiatrisch-psychotherapeutische Versorgungssituation von Patient_innen mit einer Borderline Persönlichkeitsstörung (BPS) für alle Behandlungseinrichtungen eine große therapeutische aber auch finanzielle Herausforderung dar. Mit Anteilen von $8-30 \%$ in der ambulanten und $15-20 \%$ in der stationären allgemeinpsychiatrischen Versorgung

\section{S. Margreiter ( $₫)$}

Institut für Psychotherapie mit Tageszentrum für

Borderlinestörung, Franzensbrückenstraße 5/4, 1020 Wien,

Österreich

susanne.margreiter@psd-wien.at sowie $60-80 \%$ im forensischen Bereich ist die BPS die Persönlichkeitsstörung mit der höchsten klinischen Relevanz (Bolm 2009).

Nach kurzer Darstellung der zugrundeliegenden fachlichen Konzepte wird das Therapieprogramm des Tageszentrums für Borderlinestörung (TAZ-B) vorgestellt.

Anhand von zwei Fallbeispielen werden therapeutische Prozesse und Problemstellungen dieses Krankheitsbildes verdeutlicht. Mit einem Ausblick in die Zukunft der Behandlung von Patient_innen mit Borderline Persönlichkeitsstörung schließt der Artikel.

Schlüsselwörter Ambulante psychotherapeutische Versorgung · Borderline Störung · PSD Wien · Fallbeispiele

Treatment of patients with borderline personality disorder (BPD) in a psychotherapeutic day care center in Vienna (Psychosoziale Dienste Wien)

Summary This article is about the treatment of patients suffering from borderline personality disorder (BPD) in a psychotherapeutic day care center, which was founded in 2009. The concept of our treatment is based on DBT (dialectical behavioral therapy) and lasts 12 weeks. The patients benefit from a complex well structured weekly schedule, which includes DBT-Skillstraining, psychoeducation, training of social competence, occupational therapy, sports, nutritional advice, art therapy and individual therapy. Our team consists of psychotherapists, occupational therapists and a psychiatric nurse.

The Psychosocial Services Vienna (Psychosoziale Dienste Wien) include medical help, crisis intervention and psychotherapeutic treatment.

Two case studies show how patients can develop their competences, mindfullness and wellbeing. 
Keywords Psychotherapeutic day care center · Borderline personality disorder - Psychosocial services in Vienna (PSD Wien) · Case studies

\section{Einleitung}

Seit über 15 Jahren beschäftigen wir uns am Institut für Psychotherapie mit Tageszentrum für Borderlinestörung des PSD Wien (Psychosoziale Dienste) u. a. besonders intensiv mit der Behandlung von Patient_innen mit der Diagnose „Borderline Persönlichkeitsstörung (BPS)“.

Begonnen haben wir 2002 mit einer regelmäßig stattfindenden DBT-Skillstrainingsgruppe, die schnell regen Zuspruch fand. Der zunehmende Bedarf an störungsspezifischen Therapiemöglichkeiten für BPSPatient_innen führte über die Jahre $\mathrm{zu}$ einem stetigen Ausbau und einer Weiterentwicklung unserer Behandlungsangebote. Skillstrainingsgruppen sowie Schematherapiegruppen für Patient_innen mit Borderline Störung stellen daher fixe Bestandteile unseres spezifischen psychotherapeutischen Angebotes dar.

Im Herbst 2009 wurde das Tageszentrum für Borderlinestörung (TAZ-B) als wichtige Ergänzung unserer Angebote gegründet. Längerfristige sinnvolle Tagesstruktur und intensive, störungsspezifische therapeutische Angebote sind gerade für Patient_innen mit BPS essentiell, um den Kreislauf von häufigen kurzfristigen stationären Kriseninterventionen zu durchbrechen und Schritte in Richtung von mehr Stabilität zu etablieren.

Das TAZ-B ist im ambulanten psychiatrischen Versorgungsbereich zu einer wichtigen Anlaufstelle für betroffene Patient_innen v. a. aus Wien geworden. Unsere Erfahrung zeigt, dass aber immer wieder auch Patient_innen aus den Bundesländern großes Interesse an einer Behandlung im TAZ-B haben. Vereinzelt nahmen mittlerweile Patient_innen aus fast allen Bundesländern an einem Behandlungsturnus teil. Manche Patient_innen nahmen dafür einen sehr großen organisatorischen Aufwand auf sich.

\section{Behandlungsangebot und Hintergrund}

Das Institut für Psychotherapie mit Tageszentrum für Borderlinestörung ist als Psychotherapie-Einrichtung der Psychosozialen Dienste Wien in ein ambulantes sozialpsychiatrisches Gesamtbehandlungsangebot für psychisch erkrankte Patient_innen eingebettet. Wir sind für Patient_innen mit unterschiedlichen psychiatrischen Diagnosen wie affektiven Erkrankungen, Angststörungen, Zwängen, schizophrenen Erkrankungen oder eben Persönlichkeitsstörungen zuständig. Ausschlusskriterien sind Suchterkrankungen sowie forensische Fragestellungen.

Nach einem ausführlichen Erstgespräch am Institut wird der/die Patient_in für ein spezifisches Therapieangebot vorgemerkt und zugewiesen. Nach klinischpsychologischer Abklärung können wir Einzelthera- pie, störungsspezifische Gruppenangebote oder eine Aufnahme im Tageszentrum anbieten.

Als wichtiges Kriterium für eine Behandlung am Institut für Psychotherapie sehen wir auch soziale Problemstellungen. Durch den meist frühen Beginn der Erkrankung, verfügen viele betroffenen BPS Patient_innen nicht über eine abgeschlossene Ausbildung. Der Berufseinstieg wird dadurch zusätzlich erschwert, Probleme mit der Wohnsituation oder Schulden sind dann oft die Folge. Familiäre Unterstützung ist selten gegeben, häufig fehlen positive soziale Kontakte generell. Neben Psychotherapie stellt daher auch Sozialarbeit eine wichtige Behandlungssäule dar.

Im Rahmen der Psychosozialen Dienste in Wien finden die Patient_innen in den Sozialpsychiatrischen Ambulatorien ergänzend kompetente fachärztliche Betreuung. Zur Klärung der sozialen Problematik kann dort bei Bedarf auch sozialarbeiterische Unterstützung in Anspruch genommen werden. In Krisensituationen außerhalb unserer Öffnungszeiten können sich Patient_innen an den Sozialpsychiatrischen Krisendienst des PSD wenden und professionelle Hilfe finden. Die gute Zusammenarbeit und Vernetzung mit den anderen Einrichtungen des PSD Wien ist für uns als psychotherapeutische Einrichtung äußerst bedeutsam und hilfreich.

Aufgrund dieser guten Rahmenbedingungen ließ sich für das TAZ-B ein umfassendes Betreuungsangebot, das sich an den Behandlungsrichtlinien des DBT-Konzeptes (Dialektisch Behaviorale Therapie) orientiert, realisieren.

\section{Störungsmodell}

Die Borderline-Persönlichkeitsstörung ist als eine Störung der Affektregulation sowie als Störung der sozialen Interaktionen zu verstehen. Die Ursachen der Erkrankung sind sehr komplex und bisher nicht völlig geklärt (siehe Abb. 1). Folgende empirisch gesicherte Risikofaktoren können jedenfalls zur Entwicklung einer Borderline Persönlichkeitsstörung beitragen:

- frühe (sexuelle) Gewalterfahrungen (v. a. durch enge Bezugspersonen)

- weibliche Sozialisierung

- körperliche Gewalt

- Vernachlässigung

- fehlende Sicherheit

- invalidierende Bindungserfahrungen

Ebenfalls von Bedeutung sind Genetik und Auffassungsschwierigkeiten, neurophysiologische sowie neurobiologische Funktionsstörungen der Emotionsregulation. $65 \%$ der Betroffenen wurden jedoch Opfer sexueller Gewalt, $60 \%$ erlebten körperliche Gewalt und Vernachlässigung (Bohus und Schmahl 2006).

Seit Einführung des DSM-III wird die BPS dem Cluster B der Persönlichkeitsstörungen zugeordnet (antisoziale-, Borderline-, histrionische und narziss- 
Abb. 1 Biopsychosoziales Entstehungsmodell der BPS

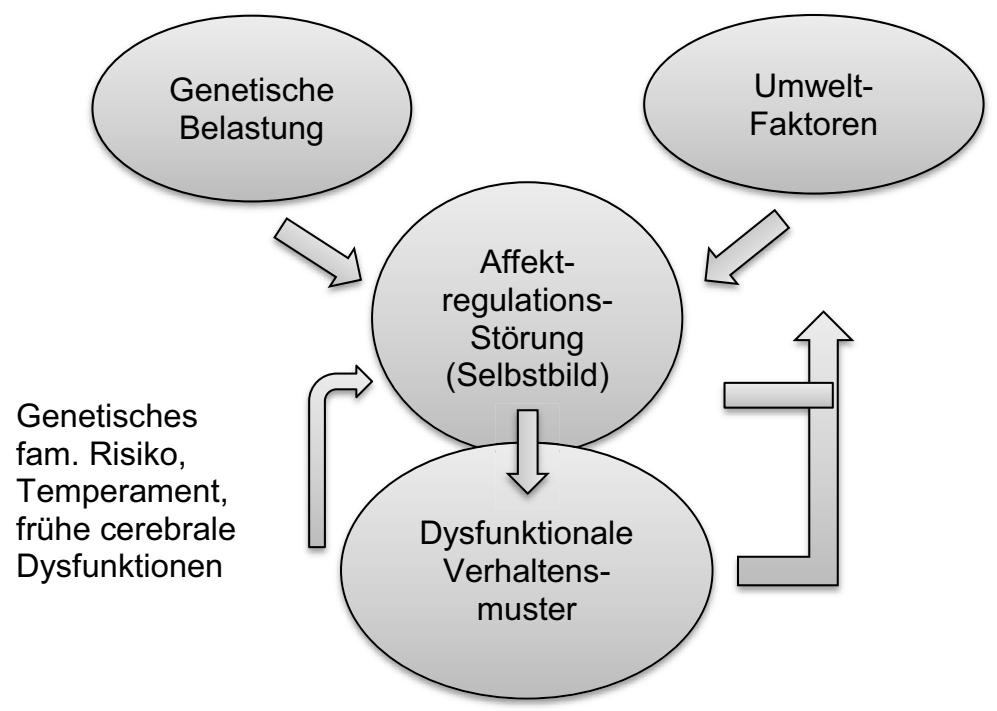

Frühe

belastende

Erfahrungen; Invalidierende

Bedingungen, BindungsErfahrungen

Kernfaktoren der Borderline Störung sind: Affektive Instabilität, Impulsivität und instabile Beziehungen.

tische Persönlichkeitsstörung). Die Diagnosestellung folgt nach klaren diagnostischen Kriterien (DSM IV bzw. ICD 10).

Viele betroffene BPS Patient_innen leiden unter zusätzlichen psychischen Störungen. Häufige komorbide Störungen sind Depressionen, Angststörungen, PTBS, Schlafstörungen aber auch Alkohol- und Drogenmissbrauch. Die Erkrankung zeigt bei Männern und Frauen unterschiedliche Verläufe. Gründe dafür mögen Unterschiede in der Sozialisation sein, oder aber auch die geschlechtsspezifische Neigung zu bestimmten Komorbiditäten. Männer nehmen seltener professionelle Hilfe in Anspruch und brechen laufende Behandlungen eher ab. Bei den Männern kommt es häufiger zu Suchtproblemen, Fremdaggressionen oder antisozialem Verhalten, Frauen leiden häufiger unter oft schweren Essstörungen und selbstverletzendem Verhalten.

Die Prävalenz der Borderline Störung in der Allgemeinbevölkerung wird je nach Studie mit 1,5 bis $\mathrm{zu}$ $3 \%$ angegeben. Damit kann von einer sehr häufigen Störung gesprochen werden. In klinischen Stichproben finden wir vermehrt betroffene Frauen. Es wird jedoch heute davon ausgegangen, dass Männer und Frauen gleich häufig von dem Störungsbild betroffen sind (vgl. Dulz et al. 2011).

\section{Psychotherapie und Borderline Störung}

Die Borderline Persönlichkeitsstörung ist heute in vielen Fällen psychotherapeutisch gut behandelbar. Dazu sind sowohl psychoanalytisch orientierte als auch verhaltenstherapeutisch orientierte störungsspezifische Methoden entwickelt worden. Die Prognose für Menschen mit der Diagnose „Borderline
Persönlichkeitsstörung“ bei störungsspezifischer psychotherapeutischer Behandlung ist daher relativ gut.

Psychotherapeutische Methoden zur Behandlung der Borderline Persönlichkeitsstörung, deren Wirksamkeit empirisch gut nachgewiesen wurden:

- Schema Focussed Therapy - ST (J. Young)

- Mentalization Based Treatment - MBT (Bateman \& Fonagy)

- Transference Focussed Psychotherapy - TFP (O. Kernberg)

- Dialektisch Behaviorale Therapie - DBT (M. Linehan)

Störungsspezifische psychotherapeutische Interventionen sind die Behandlung der Wahl bei der BPS, pharmakologische Interventionen sind als eher zweitrangig einzustufen (SGPP 2018).

Laut aktueller Studienlage gelten derzeit DBT und ST als die wirksamsten Psychotherapiemethoden in der Behandlung der Borderline Störung.

Schematherapie (ST) gilt als Weiterentwicklung kognitiv-verhaltenstherapeutischer Verfahren. Erfolgreiche Techniken der Verhaltenstherapie werden mit Elementen aus der Gestalttherapie sowie tiefenpsychologischen Sichtweisen verbunden. Die Borderline Störung entsteht nach diesem Verständnis durch ein Zusammenwirken verschiedener dysfunktionaler Funktionsweisen der Patientin oder des Patienten. In der Therapie wird auf den jeweils aktivierten Modus eingegangen. Die Ursachen von Schemata liegen häufig in traumatischen Vorerfahrungen. Ziel der Schematherapie ist es, Patient_innen Einsicht zu vermitteln, welche Bedürfnisse in der Kindheit nicht adäquat erfüllt worden sind und wie es gelingen kann, aktuelle Bedürfnisse besser zu befriedigen (vgl. Roediger 2011). 
Der Mentalisierungsansatz (MBT) stellt eine Synthese aus entwicklungspsychologischen, bindungstheoretischen, psychodynamischen, kognitiv-behavioralen, traumabezogenen sowie neurobiologischen Erkenntnissen und der „Theory of mind“ dar. Somit kann dieser Ansatz als Gemeinsamkeit zwischen allen Psychotherapieschulen gesehen werden. Mentalisieren bedeutet dabei, Verhalten im Zusammenhang mit inneren Zuständen und Vorgängen zu erleben und $\mathrm{zu}$ verstehen. Das Ziel von MBT besteht darin, dieses Verständnis zu fördern. Ein klares Konzept sowie stabile Repräsentanzen von inneren Zuständen (Gedanken, Gefühle, Wünsche, Bedürfnisse) sollten entwickelt werden (vgl. Bolm 2009).

Die Transference-Focused Psychotherapy (TFP) ist eine spezifische psychodynamische Psychotherapie, die für Patient_innen mit Cluster-B-Persönlichkeitsstörungen entwickelt wurde. Diese Methode basiert auf der Objektbeziehungstheorie und unterscheidet sich deutlich von anderen psychodynamischen Therapieansätzen. Verglichen mit klassisch psychoanalytischen Settings sind in der TFP klare Struktur und eine direkte, unterstützende Beziehungsgestaltung von größerer Bedeutung (Clarkin et al. 2001).

In der Dialektisch Behavioralen Therapie (DBT) versteht sich der/die Therapeut_in auch als „Coach“. Die DBT basiert auf überwiegend verhaltenstherapeutischen Fertigkeiten. Ergänzt wird die Therapie dabei auch durch fernöstliche Elemente. Die therapeutische Grundhaltung der DBT ist geprägt durch Akzeptanz, Stabilität, Geduld und Mitgefühl. Wichtig ist eine validierende Atmosphäre sowie Unterstützung bei der Problembewältigung. In der DBT wird die gestörte Emotionsregulation als Kernproblem der Erkrankung betrachtet. Dysfunktionales Verhalten steht in enger Verbindung mit der jeweiligen inneren Anspannung. Diese Anspannungszustände führen zu Selbstverletzungen oder anderen impulsiven Verhaltensweisen. Zwischenmenschliche Probleme treten ebenfalls vermehrt auf, da Anspannung auch zu Aggressionen führen kann. Am Beginn der Behandlung wird ein schriftlicher Behandlungsvertrag abgeschlossen, um eine gute Arbeitsgrundlage zu schaffen.

\section{DBT Methodik (vgl. Bohus 2019)}

- Dialektik zwischen Akzeptanz und Veränderung

- Problemlösetechniken

- Stabilisierungstechniken

- Kognitive Umstrukturierung

- Expositionsverfahren

- Validierungsstrategien

- Vermittlung von Skills (teaching - training - feedback)

- Bearbeitung von dysfunktionalen Schemata

Die komplexe BPS Problematik wird in der DBT nach hierarchisierten Therapiezielen bearbeitet. Die Therapieziele sind nach dem Grad der Selbstgefährdung aufgebaut. Es soll in der Therapie immer am wichtigsten Ziel gearbeitet werden:
1. Suizidalität/Selbstverletzendes Verhalten
2. Verhaltensalternativen
3. Umgang mit Emotionen
4. Lebensgestaltung

Die Behandlung erfordert von Therapeut_innen und Patient_innen Zeit, persönliches Engagement, aber auch viel Geduld und Durchhaltevermögen.

\section{Therapie im Tageszentrum für Borderlinestörung des PSD Wien}

Seit 2009 können wir im Institut für Psychotherapie mit Tageszentrum für Borderlinestörung des PSD Wien ein 12-wöchiges intensives Therapieprogramm für Patient_innen mit einer Borderline Persönlichkeitsstörung anbieten. Das Programm haben wir zunehmend adaptiert und weiterentwickelt. Unser Therapieprogramm basiert auf einem verhaltenstherapeutischen Grundkonzept und fundierten empirischen Studien der DBT.

\section{Vorrangige Ziele einer Teilnahme im Tageszentrum} (TAZ-B)

- Aktivierung von Ressourcen

- Sinnvolle Tagesstruktur

- Vermehrte Selbstkontrolle

- Steigerung der Lebensqualität

- Steigerung der Belastbarkeit

- Entwicklung von neuen Perspektiven

- Zugang zu den eigenen Gefühlen finden

- Verbesserung des Selbstwertgefühls

Störungsspezifische Behandlungselemente bei BPS nach dem DBT-Konzept (vgl. Bohus 2019)

- Einzeltherapie

- Telefonische Kontakte/Krisenintervention

- Skillstraining (16 Wochen)

- Tageszentrum (12 Wochen)

bei Bedarf:

- Fachärztliche Behandlung in den Sozialpsychiatrischen Ambulatorien

- Psychiatrische Soforthilfe

- Stationäre Aufnahme bzw. Krisenintervention an den regionalisierten psychiatrischen Abteilungen

Die Komplexität der Symptomatik dieses Erkrankungsbildes erfordert eine hohe Flexibilität des Behandlungsteams und die Bereitschaft, sich immer wieder auf neue Erfordernisse einzulassen, um das umfassende und intensive Therapieangebot anbieten zu können, das diese Patient_innen benötigen.

Unsere Patient_innen sind überwiegend weiblich (85\%), viele der männlichen Borderline-Patienten werden aufgrund ihrer Komorbiditäten, wie Suchtpro- 
Tab. 1 Elemente des Wochenplans im TAZ-B

\begin{tabular}{|c|c|c|c|c|}
\hline Montag & Dienstag & Mittwoch & Donnerstag & Freitag \\
\hline Morgenrunde & Achtsamkeits-Gruppe (PT) & Morgenrunde & Morgenrunde & Morgenrunde \\
\hline Orientierung (PT) & Ergotherapie & Skillstraining (PT) & Soziale Kompetenz (PT) & Psychoedukation (PT) \\
\hline Kunsttherapie & Wahrnehmung/ & Ernährung/Kochen & Bewegung/ & $\begin{array}{l}\text { Skillsvertiefung } \\
\text { (PT) }\end{array}$ \\
\hline Spiel und Spaß & Euthyme Verfahren (PT) & Ergotherapie & Gartengruppe & Ergotherapie \\
\hline $\begin{array}{l}\text { Einzelgespräch } \\
\text { (PT) }\end{array}$ & $\begin{array}{l}\text { Aktivierung/Einzelgespräch } \\
\text { (PT) }\end{array}$ & Sportgruppe & Kognitives Training & Wochenabschluss \\
\hline
\end{tabular}

blematik oder nach außen gerichteten Aggressionen, eher in anderen Einrichtungen betreut.

Die Teilnahme am Therapieprogramm erfolgt nach ausführlichem Erstgespräch und fundierter klinisch psychologischer diagnostischer Abklärung. Eine gewisse Motivation und Belastbarkeit sind Voraussetzung für eine erfolgreiche Teilnahme. Täglich finden fünf therapeutische Einheiten statt (siehe Tab. 1).

Im therapeutischen Team arbeiten Klinische Psycholog_innen, Psychotherapeut_innen (VT) und Ergotherapeut_innen eng zusammen.

Wirksame therapeutische Elemente sind Skillstraining, soziales Kompetenztraining, Euthyme Verfahren, Ergotherapie, Kunsttherapie, Achtsamkeit und Entspannung, Aktivierung und Sport, Ernährung sowie Psychoedukation, aber auch (Berufs)orientierung. Durch die Zusammenarbeit im gesamten Team werden die einzelnen Einheiten zu einem positiven „Ganzen“. Im Rahmen der Kunsttherapie werden beispielsweise Themen der Skillstrainingsgruppe mittels verschiedener Materialien und Medien integriert und vertieft.

Die Ergotherapie bietet Hilfestellung beim Wiedererreichen der Handlungsfähigkeit im Alltag. Defizite und Ressourcen der Patient_innen können genauer ermittelt werden, Ziele definiert und angestrebt, sowie Unterstützung bei der Ausführung von gestellten Aufgaben angeboten werden. Neue Erfahrungen $\mathrm{zu}$ machen, angenommen $\mathrm{zu}$ werden, aber auch der Austausch mit anderen Betroffenen stellen ebenfalls wichtige Elemente der Behandlung dar.

Psychotherapie findet im TAZ-B überwiegend im Gruppensetting statt. Ergänzend werden regelmäßige Einzelgespräche im Rahmen der Bezugsbetreuung angeboten.

Wenn wir Patient_innen fragen, was die Erkrankung für sie bedeutet, wird deutlich, wie stark die Beeinträchtigung in allen Lebensbereichen spürbar wird. Von ihrem Umfeld werden Betroffene häufig mit vielen Vorurteilen und Abwertungen konfrontiert. „Borderline“ bedeutet für die betroffenen Patient_innen permanenten Stress. Diese Erkrankung ist enorm anstrengend, scheinbar aus dem Nichts kann die Stimmung ins Bodenlose kippen.

\section{Borderline bedeutet für mich ...}

- Starke Müdigkeit

- Verzweiflung

- ist Energie raubend

- häufigen Realitätsverlust und Dissoziation

- Medikamente schlucken müssen

- viele Tränen

- ein Teil von mir, den ich in mein Leben integriere

- Strategien bzw. Skills lernen müssen

- sich das Leben emotional zu erschweren

- Anstrengung

- Schwarz-Weiß denken

- verständnisvoll mitfühlend, aber auch sehr verletzlich zu sein

- Alles oder Nichts - ein Dazwischen gibt es nicht

- explosionsartig aggressiv werden

\section{Was andere über Borderline denken ...}

- Hoffnungslosigkeit

- BPS Patient_innen finden keine Freunde und sind unsäglich anstrengend

- die Borderline Erkrankung ist ein Kündigungsgrund

- anders sein

- wird häufiger bei Frauen diagnostiziert

- ist meist erworben

- „verlängerte Pubertät“

- BPS heißt emotionale Mehrarbeit

- es gibt keine Heilung

- Zusammenschluss von Symptomen verschiedener Krankheitsbilder

- tritt häufig in Kombination mit anderen psychischen Erkrankungen auf

- heißt lernen damit zu leben

Anhand von zwei Fallbeispielen möchte ich abschließend einen Eindruck unserer Arbeit im TAZ-B vermitteln. Die gewählten Beispiele sollen die Vielfältigkeit unserer Teilnehmer_innen verdeutlichen.

Wir gehen ein Stück Lebensweg gemeinsam mit unseren Teilnehmer_innen und bieten ihnen die Möglichkeit, neue Erfahrungen zu machen, Erlebtes besser zu verarbeiten und gesündere Strategien für die aktuellen Herausforderungen des Lebens zu entwickeln. 


\section{Fallbeispiele}

Fallbeispiel 1 - Frau S.

Die 25-jährige Patientin wird nach einer ambulanten Rehabilitation an uns zur Weiterbehandlung im TAZ-B überwiesen. Aufgrund der vielfältigen Einschränkungen der Alltagsfunktionen wird ihre Belastbarkeit als sehr reduziert eingeschätzt. Frau S. ist bereits in psychiatrischer Behandlung und erhält TRITTICO $150 \mathrm{mg}$, SEROQUEL XR $50 \mathrm{mg}$ und ESCITALOPRAM $10 \mathrm{mg}$.

\section{Diagnosen}

- Emotional instabile Persönlichkeitsstörung, Borderline Typ (F 60.31)

- Rezidivierende Depression, mittelgradige Episode (F 33.10)

- Chronische Schmerzsymptomatik (F 45.4)

Im ausführlichen Erstgespräch beschreibt die Patientin ihre Kindheit in der Slowakei, die von massiver Gewalt durch die Eltern und chronisch invalidierende Bedingungen gekennzeichnet war. Ab dem 15. LJ war Frau S. auf sich alleine gestellt. Die erlebten Belastungen und die schwere Vernachlässigung führten bereits in ihrer frühen Jugend zu selbstverletzendem Verhalten und schließlich zu einem schweren Suizidversuch durch Tablettenintoxikation mit nachfolgendem längeren stationärem Aufenthalt.

Aufgrund ihrer psychischen Problematik war die Patientin nicht in der Lage die Schule erfolgreich abzuschließen. Um zu überleben, musste sie sich im Rotlicht-Milieu und in der Drogenszene ihr Geld verdienen. Auch in dieser Zeit kam es zu vielen traumatisierenden Erfahrungen. Ab dem 20. LJ lebt Frau S. in Wien. Auch hier hat sie bereits viele negative Beziehungserfahrungen gemacht und wurde von ihren Partnern schwer enttäuscht und betrogen.

Frau S. reagierte immer wieder verstärkt mit körperlichen Symptomen wie Fieberschüben, Lähmungserscheinungen der Beine sowie Schmerzen am ganzen Körper. Sie reagierte schnell impulsiv und aggressiv.

\section{Therapieziele}

- Soziale Fertigkeiten verbessern

- Arbeit am Selbstwert

- „mutiger“ werden

- Emotionskontrolle verbessern

- Interessen (wieder)entdecken

Anfangs fiel es der Patientin im TAZ-B sehr schwer, ein wenig Vertrauen in Therapeut_innen und das Therapieangebot zu entwickeln. Auch aufgrund sprachlicher Probleme hatte sie Hemmungen, sich in die Gruppe einzubringen. Es gelang uns erst Schritt für Schritt besser mit der Patientin in Kontakt zu kommen und auch ihre humorvolle Seite kennenzulernen.
Frau S. entwickelte sich über die 12 Wochen $\mathrm{zu}$ einer verlässlichen, liebenswerten und bemühten Teilnehmerin. Einen Ort zu haben, an dem sie willkommen und sicher war, war wichtig. Sie war eine Kämpferin, die trotz ihrer häufig starken Schmerzsymptomatik, sehr interessiert und engagiert war. Frau S. konnte ihre Therapieziele überwiegend erreichen bzw. Schritte in eine konstruktive Richtung machen. Soziales Kompetenztraining und die Skillstrainingsgruppe waren besonders hilfreich und ermöglichten ihr, neue Fertigkeiten zu erwerben, diese anzuwenden und dadurch neue Erfahrungen zu machen. Als große Ressource erlebte sie ihren wiederentdeckten Zugang zu ihrer Kreativität. Durch die Ergotherapie konnte sie sich verschiedene neue Techniken aneignen, die ihr große Freude bereiteten und ihren Selbstwert steigerten.

Ihre finanzielle und soziale Situation war auch am Ende ihrer Teilnahme leider weiter sehr angespannt. Ein Kontakt mit der Schuldnerberatung wurde mit unserer Unterstützung hergestellt. Frau S. hat um Rehabilitationsgeld angesucht, um eine gewisse finanzielle Absicherung zu erreichen. In ihrem Umfeld gab es leider wenig unterstützende, konstruktive Kontakte. Sie konnte sich jedoch von einigen destruktiven Personen besser abgrenzen und sich mehr auf ihre eigenen Ziele konzentrieren.

Frau S. blieb auch nach ihrem Therapieabschluss im TAZ-B mit uns in Kontakt und kam regelmäßig in die Sportgruppe, die sich auch als Angebot für ehemalige Teilnehmer_innen versteht.

\section{Fallbeispiel 2 - Frau T.}

Frau T., 47 Jahre alt, meldet sich auf Empfehlung der ÖGK (Österreichische Gesundheitskasse) bei uns und ersucht um psychotherapeutische Unterstützung. Die Patientin wird nach dem Erstgespräch primär für eine Einzeltherapie vorgemerkt, die sie nach einer kurzen Wartezeit beginnen kann. Aufgrund ihrer aktuell sehr schwierigen Lebenssituation und einer sich abzeichnenden massiveren psychischen Krise wird Frau T. zusätzlich für eine Teilnahme am TAZ-B vorgemerkt. Die Patientin ist seit ihrer Jugend immer wieder auch in psychiatrischer Behandlung bei einem niedergelassenen Facharzt und erhält aktuell CIPRALEX $10 \mathrm{mg}$ und ATARAX $25 \mathrm{mg}$.

\section{Diagnosen}

- Emotional instabile Persönlichkeitsstörung, Borderline Typ (F 60.31)

- Rezidivierende Depression, mittelgradige Episode (F 33.10)

- Binge Eating Disorder (F 50.9)

Frau T. wuchs gemeinsam mit ihrem jüngeren Bruder in ihrer Familie im Burgenland in einer kleinen Ortschaft auf. Die Eltern führten eine schwierige Ehe, Frau T. wurde bereits in jungem Alter zur Vertrauten 
und Komplizin der Mutter. Wirklich geliebt gefühlt habe sie sich nicht, jedoch versuchte sie mit großen Anstrengungen immer Aufmerksamkeit und Zuwendung von den Eltern zu bekommen. Der Vater habe sich in andere Beziehungen geflüchtet, Frau T. wurde häufig zur Geheimnisträgerin. Aussehen spielte in der Familie eine große Rolle, v. a. auch schlank zu sein. Sie sei häufig abgewertet und belächelt worden, obwohl sie eigentlich nie „zu dick“ gewesen war. Die Mutter litt häufig unter Depressionen und Migräne. Sie konnte sich dadurch nicht um die Bedürfnisse der Kinder kümmern. Die Patientin übernahm früh die Verantwortung für den jüngeren Bruder, der als Sohn der Familie einen anderen, besseren Stellenwert einnahm.

Die Ausbildungswahl fiel der Patientin schwer. $\mathrm{Zu}$ wissen, was sie wolle und an sich selbst zu glauben, war durch das stark reduzierte Selbstwertgefühl erschwert. Frau T. begann verschiedene Berufsausbildungen, schloss jedoch keine Lehre ab.

Sie flüchtete sich früh in die Ehe mit einem Italiener und lebte in der Folge fast 20 Jahre im Ausland. In der Beziehung mit ihrem Mann setzt sich der Leidensweg von Frau T. fort. Er war dominant, wenig wertschätzend oder unterstützend. Die Patientin versuchte für ihre drei Kinder eine gute Mutter zu sein, litt jedoch immer wieder unter depressiven Phasen und suchte über viele Jahre häufig auch therapeutische Hilfe auf. Den Kindern zuliebe wollte sie sich lange nicht scheiden lassen. Sie flüchtete sich in destruktive Verhaltensweisen wie übermäßiges Essen, Selbstbestrafung durch selbstauferlegte Verbote oder dissoziative Tagträume. Behandelt wurde die Patientin bisher ausschließlich aufgrund ihrer Depressionen.

Nach der Scheidung kam Frau T. mit 40 Jahren wieder nach Österreich zurück. Sie habe sich hier bessere Berufschancen erhofft. Ihre beinahe erwachsenen Kinder blieben in Italien, Frau T. leidet sehr unter der räumlichen Distanz. Die Eingewöhnung wieder in Österreich fiel der Patientin schwer. Sie tat sich schwer, eine Arbeit $\mathrm{zu}$ finden und fühlte sich alleine in ihrer Wohnung einsam. Seither kam es immer wieder zu massiven psychischen Krisen, die zum Teil auch stationäre Aufenthalte zur Krisenintervention erforderten. Sie war arbeitslos und fühlte sich vom Druck des AMS überfordert. Frau T. konnte sich jedoch auch nicht vorstellen, wieder arbeiten zu gehen.

Bei Beginn der Psychotherapie am Institut war die Patientin psychisch sehr instabil. Sie konnte sich nicht gut konzentrieren, litt unter reduziertem Selbstwert und ausgeprägten Versagensängsten. Sie fühlte sich hoffnungslos, resignativ und unproduktiv. Auch schlief sie viel und aß sehr unregelmäßig.

Die durchgeführte klinisch psychologische Testung bestätigte die Diagnosen emotional instabile Persönlichkeitsstörung vom Borderline Typ (F 60.31) sowie eine rezidivierende Depression, die zum aktuellen Zeitpunkt in mittelgradiger Ausprägung (F 33.10) vorhanden war. Auch eine Bing Eating Störung (F 50.9) wurde festgestellt.
Im TAZ-B gehörte die Patientin zu den älteren Teilnehmer_innen. Das Durchschnittsalter der Teilnehmer_innen liegt bei ca. 27 Jahren. Frau T. konnte sich dennoch gut in die bestehende Gruppe einfügen und profitierte von dem Austausch und der Zusammenarbeit mit den anderen Gruppenmitgliedern.

Anfangs geriet die Patientin immer wieder in depressive Krisen, konnte die Gesprächsangebote durch ihre Bezugsbetreuerin jedoch gut annehmen und Hilfestellungen aufgreifen. Wichtig im therapeutischen Prozess waren für sie v.a. eine Realitätsüberprüfung ihrer aktuellen Emotionen und Wahrnehmungen, ihr Selbstmitgefühl zu stärken, der Umgang mit Selbstkritik und Schamgefühlen, Verbesserung der Spannungsregulation sowie Fokussieren ihrer Stärken und Qualitäten (vgl. Diedrich 2016).

Frau T. hatte große Widerstände gegenüber den handwerklichen Angeboten im Programm des TAZ-B. Sie schaffte es, diese zu überwinden und sich an allen Gruppenangeboten konstruktiv zu beteiligen. Heute ist sie im Nachhinein sehr stolz auf die Werkstücke, die in diesen Einheiten entstanden sind.

Die Einzeltherapie wurde nach Abschluss des TAZB-Turnus fortgesetzt. Auch jetzt in der Corona-Krisenzeit ist die Patientin bemüht, weiter an ihren Themen und Schwierigkeiten zu arbeiten. Sie schafft es, meist konstruktiv mit sich umzugehen und auch schlechtere Tage zu bewältigen, ohne in ihre „alten Muster“ zurückzufallen. Die therapeutische Beziehung zu ihr ist stabil und tragfähig. Frau T. hat gute Ziele, die sie nach einer Normalisierung der aktuellen allgemeinen Situation anstreben wird.

\section{Ausblick}

Seit September 2009 haben ca. 500 Patient_innen an einem therapeutischen Aufenthalt im TAZ-B teilgenommen. Für viele Teilnehmer_innen war es ein wichtiger Schritt auf dem Weg in Richtung mehr Perspektive, Gesundheit, Lebensqualität und Stabilität.

Borderline Patient_innen benötigen viel Halt, Verlässlichkeit, Beziehung und Stabilität. Sehr häufig fehlen diesen Patient_innen stabile, authentische, zwischenmenschliche Beziehungen in ihrem privaten Kontext. Viele unserer Patient_innen tun sich durch erlebte invalidierende Bedingungen in der Kindheit sehr schwer, Vertrauen zu anderen Menschen aufzubauen. Ein Stück Wertschätzung und Akzeptanz im Kontext des Behandlungsangebotes im TAZ-B zu erleben, sehe ich als große Chance und als wichtige therapeutische Aufgabe.

Interessenkonflikt S. Margreiter gibt an, dass kein Interessenkonflikt besteht.

Open Access Dieser Artikel wird unter der Creative Commons Namensnennung 4.0 International Lizenz veröffentlicht, welche die Nutzung, Vervielfältigung, Bearbeitung, Verbreitung und Wiedergabe in jeglichem Medium und Format erlaubt, sofern Sie den/die ursprünglichen Autor(en) und die Quelle 
ordnungsgemäß nennen, einen Link zur Creative Commons Lizenz beifügen und angeben, ob Änderungen vorgenommen wurden.

Die in diesem Artikel enthaltenen Bilder und sonstiges Drittmaterial unterliegen ebenfalls der genannten Creative Commons Lizenz, sofern sich aus der Abbildungslegende nichts anderes ergibt. Sofern das betreffende Material nicht unter der genannten Creative Commons Lizenz steht und die betreffende Handlung nicht nach gesetzlichen Vorschriften erlaubt ist, ist für die oben aufgeführten Weiterverwendungen des Materials die Einwilligung des jeweiligen Rechteinhabers einzuholen.

Weitere Details zur Lizenz entnehmen Sie bitte der Lizenzinformation auf http://creativecommons.org/licenses/by/4. $0 /$ deed.de.

\section{Literatur}

\section{Verwendete Literatur}

Bohus, M. (2019). Borderline Störung. Fortschritte der Psychotherapie. Göttingen:Hogrefe.

Bohus, M., \& Schmahl, C. (2006). Psychopathologie und Therapie der Borderline-Persönlichkeitsstörung. Deutsches Ärzteblatt, 103(49), A3345-A3352.

Bolm, T. (2009). Mentalisierungsbasierte Therapie (MBT) für Borderline Störungen und chronifizierte Traumafolgen. Köln:Deutscher Ärzte-Verlag.

Clarkin, J., Yeomans, F., \& Kernberg, O. (2001). Psychotherapie der Borderline-Persönlichkeit. Manual zur TransferenceFocused Psychotherapy (TFP). Stuttgart: Schattauer.

Diedrich, A. (2016). Mitgefühlsfokussierte Interventionen in der Psychotherapie. Göttingen:Hogrefe.

Dulz, B., Herpetz, S., Kernberg, O., \& Sachsse, U. (2011). Handbuch der Borderline-Störungen. Stuttgart: Schattauer.
Roediger, E. (2011). Praxis der Schematherapie. Lehrbuch zu Grundlagen, Modell und Anwendung. Stuttgart: Schattauer.

SGPP Behandlungsempfehlungen Borderline-Persönlichkeitsstörung (2018)

\section{Weiterführende Literatur}

American Psychiatric Association (2000). Diagnostic and statistical manual of mental disorders (DSM-IV-TR) (4. Aufl.). Washington, DC: American Psychiatric Press. dt. Version und Einführung: Saß H, Wittchen HU, Zaunig M (2003) Diagnostisches und statistisches Manual PsychischerStörungen DSM-IV-TR. Hogrefe, Göttingen, Bern, Toronto, Seattle

Bohus, M., \& Wolf, M. (2009). Interaktives Skillstraining für Borderline-Patienten. Stuttgart: Schattauer.

Fiedler, P. (2007). Persönlichkeitsstörungen. Weinheim: Beltz.

Fleischhaker, C., \& Schulz, E. (2010). Borderline Persönlichkeitsstörungen im Jugendalter. Berlin Heidelberg: Springer.

Jacob, G., \& Arntz, A. (2011). Schematherapie in der Praxis. Weinheim, Basel: Beltz.

Kröger, Ch , \& Unckel, Ch (Hrsg.). (2006). Borderline-Störung. Wie mir die dialektisch-behaviorale Therapie geholfen hat. Göttingen: Hogrefe.

Linehan, M. (1996). Dialektisch-Behaviorale Therapie der Borderline-Persönlichkeitsstörung. München: CIP-Medien.

Schoppmann, S., Herrmann, M., \& Tilly, Ch (2019). Borderline begegnen. Miteinander umgehen lernen. Köln:Psychiatrie Verlag Gmbh.

Young, J., Klosko, J., \& Weishaar, M. (2003). Schematherapie ein praxisorientiertes Handbuch. Verlag: Junfermann.

Hinweis des Verlags Der Verlag bleibt in Hinblick auf geografische Zuordnungen und Gebietsbezeichnungen in veröffentlichten Karten und Institutsadressen neutral. 